\title{
INVERSE POWER LAW POTENTIALS ABOUT POLYGONAL PRISMS AND IN POLYGONAL CAVITIES
}

\author{
J. R. PHILIP
}

(Received 21 July 1977)

(Revised 9 January 1978)

\begin{abstract}
The effects on adsorption of the geometry of the solid may be studied through calculations based on a (distance) ${ }^{-\varepsilon}(\varepsilon>3)$ intermolecular potential. This paper establishes the result that the potential due to an infinitely long polygonal homogeneous solid prism, at position $\mathbf{r}$ in the plane of its right section, is $-\frac{1}{2} \sum_{i=1}^{n} \rho_{i}^{3-\varepsilon} \sum_{j=1}^{2} g\left(\varepsilon ; \theta_{i j}\right)$. Here $\rho_{i}=\left|\mathrm{r}-\mathrm{r}_{i}\right|$, where the $\mathbf{r}_{i}$ are the position vectors of the $n$ vertices of the polygon, and $\theta_{i j}$ are the angles $\mathbf{r}-\mathbf{r}_{i}$ makes with the two sides of the polygon which meet at vertex $\mathbf{r}_{i}$. The $g$ 's are exact functions of $\theta_{i j}$. They are, in general, integrals of associated Legendre functions, but they are elementary for $\varepsilon$ an even integer. A similar result holds for the potential within an infinitely long polygonal prismatic cavity. The analysis involves a systematic superposition schema and the concept of a supplementary potential with datum within the solid at infinity. The cases $\varepsilon=6$ and $\varepsilon=4$ are treated in detail and illustrative solutions given for the following configurations: semi-infinite laminae, deep rectangular cracks, square prisms, square prismatic cavities and regular n-gonal prismatic cavities.
\end{abstract}

\section{Introduction}

There is need for much further study "to establish quantitatively the way in which adsorption by solid bodies is dependent upon their separation and orientation" [3]. A recent unitary approach to capillary condensation and adsorption [6] has enabled investigation of the interaction between these processes for a number of configurations of the solid. The central concept of the unitary approach is that the equilibrium liquid-vapour interface is a surface of constant partial specific Gibbs free energy, $\Phi=\Phi_{*}$, which satisfies an appropriate differential equation. Here $\Phi_{*}$ is the sum of the capillary potential, $C$, dependent on the mean curvature of the 
interface, and the adsorptive potential, $\Psi$. For an effectively infinite plane solid surface, $\Psi$ depends only on $\nu$, the normal distance from the surface to the interface (the "film thickness"), but in general $\Psi$ depends in a more complicated way on the configuration of the interface relative to the solid. To evaluate the influence of geometry on adsorption (that is on $\Psi$ ), we need to calculate the adsorptive force field for various configurations of the solid.

We may do this by integrating the intermolecular potential over the solid volume. An appropriate form of the intermolecular potential is

$$
d \Psi=h(\rho) d V,
$$

where $d \Psi$ is the potential of the force field exerted by volume element $d V$ of the solid at a point at distance $\rho$ from it. Philip [7] showed that $h(\rho)$ could be inferred from experimental data on $\Psi(v)$ through the relation

$$
h(\rho)=\pi^{-1} d /\left.d \nu^{2}[d \Psi / d \nu]\right|_{\nu=\rho} .
$$

We work, in particular, with the inverse power law form

$$
h(\rho)=-\gamma \rho^{-\varepsilon}, \varepsilon>3,
$$

with $\gamma$ and $\varepsilon$ positive constants. We give special attention to the cases $\varepsilon=6$ and $\varepsilon=4$. To simplify some equations we write $\mu$ for $\pi \gamma / 6$ when $\varepsilon=6$ and $\lambda$ for $\pi \gamma$ when $\varepsilon=4$.

Steele and Halsey [10] made the first relevant study. Using (3) with $\varepsilon=6$, that is the inverse power law of London, they evaluated $\Psi$ between parallel plates and gave an integral expression for $\Psi$ in circular tubes. Van der Waals or dispersion forces are approximated by $\varepsilon=6$, but electrostatic and other forces are involved also in adsorption. See, for example, the reviews by Sheludko [9] and Clifford [1]. Philip $[6,7]$ found that experimental data on adsorbed water films in the relative humidity range 0.70 to 0.99 could be empirically fitted by taking $\varepsilon=4$. He obtained exact solutions $(\varepsilon>3)$ for the following configurations [7]: parallel plates, tubes, solid cylinders, spherical cavities, solid spheres, wedge-shaped pores and solid edges.

It was noticed that superposition of various solutions for wedge configurations would enable evaluation, in exact closed form, of inverse power law potentials about all homogeneous solid polygonal prisms of effectively infinite length, and also inside analogous polygonal prismatic cavities within a homogeneous solid. In this paper we develop a systematic superposition schema which yields general results embracing the exact solutions to all such problems.

\section{Potential outside a solid double wedge}

Our point of departure is a variant on the solution given in equations (39)-(41) of [7]. Using cylindrical coordinates $(r, \theta, z)$, we take planes $\theta=\pi-\beta, \pi, 2 \pi-\beta, 2 \pi$ 
as the surfaces of the solid double wedge with apical angle $\beta$. The regions $\pi-\beta \leqslant \theta \leqslant \pi, 2 \pi-\beta \leqslant \theta \leqslant 2 \pi$, are occupied by solid. The appropriate integral of $(1),(3)$ is

$$
\Psi(\varepsilon ; r, \theta)=-\gamma \int_{-\infty}^{\infty}\left[\int_{\pi-\beta}^{\pi}+\int_{2 \pi-\beta}^{2 \pi}\right] \int_{0}^{\infty}\left[r_{0}^{2}-2 r r_{0} \cos \left(\theta_{0}-\theta\right)+r^{2}+z^{2}\right]^{-\epsilon / 2} r_{0} d r_{0} d \theta_{0} d z
$$

Integration with respect to $z$ is elementary and integration with respect to $r_{0}$ involves associated Legendre functions [2], which it is convenient to express in terms of the hypergeometric function, $\mathbf{F}[, ; ; ;]$. We thus obtain

$$
\Psi(\varepsilon ; r, \theta)=-r^{3-\varepsilon}\left[\int_{\pi-\beta-\theta}^{\pi-\theta}+\int_{2 \pi-\beta-\theta}^{2 \pi-\theta}\right] G\left(\varepsilon ; \theta_{0}\right) d \theta_{0}
$$

with

$$
G(\varepsilon ; \theta)=-\gamma \frac{\pi^{\frac{1}{2}} \Gamma\left[\frac{1}{2}(\varepsilon-3)\right]}{2(\varepsilon-2) \Gamma\left(\frac{1}{2} \varepsilon\right)} \frac{1}{2}(1-\cos \theta)^{1-\frac{1}{2} \varepsilon} \mathbf{F}\left[\frac{1}{2} \varepsilon-2,3-\frac{1}{2} \varepsilon ; \frac{1}{2} \varepsilon ; \frac{1}{2}(1+\cos \theta)\right] .
$$

The final two factors of the right-hand side of (6) may be written more simply (though not more usefully for present purposes) as

$$
\mathbf{F}\left[2, \varepsilon-3 ; \frac{1}{2} \varepsilon ; \frac{1}{2}(1+\cos \theta)\right] \text {. }
$$

Let $\theta_{1}, \theta_{2}$ be the angles made by any plane $\theta(0<\theta<\pi-\beta, \pi<\theta<2 \pi-\beta)$ with the two adjacent solid surfaces. Then, in view of symmetry, we may write the integral in (5) as

$$
\left[\int_{\theta_{1}}^{\theta_{1}+\beta}+\int_{\theta_{2}}^{\theta_{2}+\beta}\right] G\left(\varepsilon ; \theta_{0}\right) d \theta_{0} .
$$

Also, since $\beta=\pi-\theta_{1}-\theta_{2}$, the integrals become $\int_{\theta_{1}}^{\pi-\theta_{2}}+\int_{\theta_{2}}^{\pi-\theta_{1}}$, that is, $\int_{\theta_{1}}^{\pi-\theta_{1}}+\int_{\theta_{2}}^{\pi-\theta_{2}}$. It follows that (5) is of the form

where

$$
\Psi(\varepsilon ; r, \theta)=-r^{3-\varepsilon} \sum_{j=1}^{2} g\left(\varepsilon ; \theta_{j}\right),
$$

$$
0<\theta_{j}<\pi, \quad g\left(\varepsilon ; \theta_{j}\right)=\int_{\theta_{j}}^{\pi-\theta_{j}} g(\varepsilon ; \theta) d \theta .
$$

We now relax restriction of $\theta_{j}$ to values less than $\pi$ by redefining the $\theta_{j}$ as the angles the plane $\theta$ makes with the two solid surfaces, taken directed outward from the solid. It is readily shown then that when one or both $\theta_{j}$ are taken as reflex, the foregoing equation (7) still holds with

$$
\pi<\theta_{j}<2 \pi, \quad g\left(\varepsilon ; \theta_{j}\right) \equiv g\left(\varepsilon ; \theta_{j}-\pi\right)=\int_{\theta_{j}-\pi}^{2 \pi-\theta_{j}} g(\varepsilon ; \theta) d \theta .
$$

For $\varepsilon>2, \mathbf{F}\left[\frac{1}{2} \varepsilon-2,3-\frac{1}{2} \varepsilon ; \frac{1}{2} \varepsilon ; \frac{1}{2}(1+\cos \theta)\right]$ is real, positive and finite in $0 \leqslant \theta \leqslant \pi$. The one singularity in $G(\theta)$ in $0 \leqslant \theta \leqslant \pi$ arises therefore from the term 
$\left[\frac{1}{2}(1-\cos \theta)\right]^{1-\frac{1}{2} \varepsilon}$, which behaves like $\theta^{2-\varepsilon}$ as $\theta \rightarrow 0+$. For $\varepsilon \geqslant 3, g(\varepsilon ; \theta)$, which in view of (8) is an odd function of $\theta-\frac{1}{2} \pi$, approaches $+\infty$ as $\theta \rightarrow 0+$ and approaches $-\infty$ as $\theta \rightarrow \pi-$; and, in view of (9), it approaches $+\infty$ as $\theta \rightarrow \pi+$ and approaches $-\infty$ as $\theta \rightarrow 2 \pi-$.

When $\varepsilon$ is an even integer $(\geqslant 4), F$ reduces to a polynomial of finite degree and integration of $G$ to secure $g$ is elementary. We thus find that

$$
\begin{aligned}
& \Psi(6 ; r, \theta)=-\mu r^{-3} \sum_{j=1}^{2}\left[\cot ^{3} \theta_{j}+\frac{3}{2} \cot \theta_{j}\right], \\
& \Psi(4 ; r, \theta)=-\lambda r^{-1} \sum_{j=1}^{2} \cot \theta_{j} .
\end{aligned}
$$

It will be noted that $g(6 ; \theta)$ and $g(4 ; \theta)$ exemplify the behaviour of $g(\varepsilon ; \theta)$ deduced above.

\section{Inside a solid double wedge: the supplementary potential $\Psi^{*}$}

For our present purposes we require also to evaluate the distribution of potential inside the double wedge, that is in the regions $\pi-\beta<\theta<\pi, 2 \pi-\beta<\theta<2 \pi$. In this connection we encounter the difficulty that, in these $\theta$-ranges, the integral in (4) is divergent. In physical terms, this arises because the work done by the adsorptive field on a test particle which moves across the wedge surface from outside the solid to inside it is infinite.

We may, nevertheless, introduce a potential which is finite and well defined in the regions $\pi-\beta<\theta<\pi, 2 \pi-\beta<\theta<2 \pi$. We define the supplementary potential $\Psi^{*}(\varepsilon ; r, \theta)$ as the work per unit mass required to move a test particle from a point at infinity within the solid double wedge [that is from $\left(\infty, \theta^{*}\right)$, with $\pi-\beta<\theta^{*}<\pi$ or $2 \pi-\beta<\theta^{*}<2 \pi$ ] to the point $(r, \theta)$. [The ordinary potential $\Psi(\varepsilon ; r, \theta)$ is of course the work per unit mass required to move a test particle from a point at infinity outside the solid.]

We observe that the case $\beta=\pi$ corresponds to occupation of the whole of three-dimensional space by the solid. In this case the net force on the test particle, for all $(r, \theta)$, is zero, so that

$$
\Psi_{\pi}^{*}(\varepsilon ; r, \theta) \equiv 0 .
$$

Here, and in what follows, the suffix to $\Psi^{*}$ denotes the value of the apical angle.

We may now evaluate $\Psi_{\beta}^{*}$ for $0<\beta<\pi$. In this case the solid double wedge is equivalent to solid three-dimensional space minus a second solid double wedge $0<\theta<\pi-\beta, \pi<\theta<2 \pi-\beta$. Accordingly, the supplementary potential $\Psi_{\beta}^{*}$ is equal to $\Psi_{\pi}^{*}$ minus the work per unit mass required to move the particle from $\left(\infty, \theta^{*}\right)$ to $(r, \theta)$ against the force field due to the second solid double wedge. Since $\Psi_{\pi}^{*} \equiv 0$, 
we find, analogous to (5),

$$
\Psi *(\varepsilon ; r, \theta)=r^{3-\varepsilon} \sum_{j=1}^{2} g\left(\varepsilon ; \vartheta_{j}\right)
$$

where $\vartheta_{1}, \vartheta_{2}$ are the angles the plane $\theta$ makes with the two adjacent solid surfaces of the second double wedge. If we now write $\theta_{j}$ for either $\pi-\vartheta_{j}$ or $2 \pi-\vartheta_{j}$, (13) becomes

$$
\Psi^{*}(\varepsilon ; r, \theta)=-r^{3-\varepsilon} \sum_{j=1}^{2} g\left(\varepsilon ; \theta_{j}\right)
$$

since $-g(\varepsilon ; \theta)=g(\varepsilon ; \pi-\theta)=g(\varepsilon ; 2 \pi-\theta)$. We observe that the expressions (14) for $\Psi^{*}$ and (7) for $\Psi$ are identical; and we note further the consistent definition of $\theta_{j}$ which holds in both (7) and (14), namely that the $\theta_{j}$ are the angles the plane $\theta$ makes with the two solid surfaces, taken directed outward from the solid.

\section{Potential inside a polygonal prismatic cavity}

Consider now an arbitrary polygonal prismatic cavity, infinite in the $z$-direction, and surrounded by homogeneous solid occupying the remainder of threedimensional space. On any plane $z=$ constant, the cross-section of the cavity is the $n$-gon defined by the $n$ vertices with position vectors $\mathbf{r}_{i}(i=1,2, \ldots, n)$. We proceed to establish a general expression for the potential within this cavity when the inverse power law (3) holds.

In view of Theorem 2 of the Appendix, superposition of the double wedges associated with the exterior angles of the $n$-gon doubly fills three-dimensional space, other than the cavity, which is left empty.

The potential within the cavity due to the surrounding solid is therefore exactly half that due to this superposition of double wedges. It therefore follows from (7) that within this cavity,

$$
\Psi(\varepsilon ; \mathbf{r})=-\frac{1}{2} \sum_{i=1}^{n} \rho_{i}^{3-\varepsilon} \sum_{j=1}^{2} g\left(\varepsilon ; \theta_{i j}\right)
$$

Here $\mathbf{r}$ is the position vector of the point considered in the plane $z=$ constant, $\rho_{i}=\left|\mathbf{r}-\mathbf{r}_{i}\right|$, and $\theta_{i j}$ are the angles $\mathbf{r}-\mathbf{r}_{i}$ makes with the two sides of the $n$-gon which meet at the vertex $\mathbf{r}_{i}$. The $\theta_{i j}$ are outward directed from the solid surface.

It follows, similarly that the supplementary potential at a point in the surrounding solid is exactly half the sum of the supplementary potentials due to the two double wedges which include the point and of the potentials due to the $(n-2)$ double wedges which exclude the point. We therefore have, from (14) and (7), that within the surrounding solid,

$$
\Psi *(\varepsilon ; \mathbf{r})=-\frac{1}{2} \sum_{i=1}^{n} \rho_{i}^{3-e} \sum_{j=1}^{2} g\left(\varepsilon ; \theta_{i j}\right)
$$


From (10) and (11) we have the special forms of (15) and (16):

within the cavity,

within the surrounding solid, $\Psi *(6 ; \mathbf{r})=-\frac{\mu}{2} \sum_{i=1}^{n} \rho_{i}^{-3} \sum_{j=1}^{2}\left(\cot ^{3} \theta_{i j}+\frac{3}{2} \cot \theta_{i j}\right)$,

within the cavity,

Within the surrounding solid, $\left.\begin{array}{c}\Psi(4 ; \mathbf{r}) \\ \text { W }(4 ; \mathbf{r})\end{array}\right\}=-\frac{\lambda}{2} \sum_{i=1}^{n} \rho_{i}^{-1} \sum_{j=1}^{2} \cot \theta_{i j}$.

\section{Potential about a polygonal prism}

The general expression for the potential about a homogeneous solid prism of arbitrary polygonal cross-section, infinite in the $z$-direction, follows from (16). We denote by $\Psi(\varepsilon ; \mathbf{r})_{\text {prism }}$ the potential due to the solid prism at point $\mathbf{r}$ outside it; and we denote by $\Psi^{*}(\varepsilon ; \mathbf{r})_{\text {cavity }}$ the supplementary potential at the same point due to the homogeneous solid occupying all three-dimensional space less the space occupied by the prism. We then have

$$
\begin{array}{r}
\underset{\text { prism }}{\Psi(\varepsilon ; \mathbf{r})}+\underset{\text { cavity }}{\Psi^{*}(\varepsilon ; \mathbf{r})}=\Psi_{\pi}^{*}(\varepsilon ; \mathbf{r}) \equiv 0, \\
\underset{\text { prism }}{\Psi(\varepsilon ; \mathbf{r})}=-\underset{\text { cavity }}{\Psi *}(\varepsilon ; \mathbf{r}) .
\end{array}
$$

It then follows from (16) and (19) that

$$
\text { outside prism, } \Psi(\varepsilon ; \mathbf{r})=-\frac{1}{2} \sum_{i=1}^{n} \rho_{i}^{8-8} \sum_{j=1}^{2} g\left(\varepsilon ; \theta_{i j}\right) .
$$

Note that the $\theta_{i j}$ for the prism correspond to $2 \pi-\theta_{i j}$ for the cavity. The negative sign on the right of $(20)$ follows, since $-g(\varepsilon ; \theta)=g(\varepsilon ; 2 \pi-\theta)$.

The required expression for the potential is thus identical with (15); and the special forms of (15), such as the first of (17) and (18), carry over to the prism. Although the symbols have the same meaning as in Section 4, the configurations are, of course, totally different.

\section{Illustrative examples}

We illustrate the general results of Sections 3 and 4 with a few simple examples.

\subsection{Semi-infinite laminae}

We first consider the potential about semi-infinite laminae. Taking Cartesian coordinates $(x, y, z)$, let the solid occupy the region $x \geqslant 0,-Y \leqslant y \leqslant Y$. Equation (20) immediately yields the required solution, with the first of (17) and (18) giving the 
following particular results:

$$
\begin{aligned}
\Psi(6 ; x, y)= & -\frac{\mu}{2}\left\{\left[x^{2}+(y-Y)^{2}\right]^{-3 / 2}\left[\left(\frac{x}{y-Y}\right)^{3}+\frac{3}{2}\left(\frac{x}{y-Y}+\frac{y-Y}{x}\right)+\left(\frac{y-Y}{x}\right)^{3}\right]\right. \\
& -\left[x^{2}+(y+Y)^{2}\right]^{-3 / 2}\left[\left(\frac{x}{y+Y}\right)^{3}+\frac{3}{2}\left(\frac{x}{y+Y}+\frac{y+Y}{x}\right)+\left(\frac{y+Y}{x}\right)^{3}\right] \\
& \left.+(y-Y)^{-3}-(y+Y)^{-3}\right\} ; \\
\Psi(4 ; x, y)= & -\frac{\lambda}{2}\left\{\left[x^{2}+(y-Y)^{2}\right]^{-1 / 2}\left[\frac{x}{y-Y}+\frac{y-Y}{x}\right]\right. \\
& \left.-\left[x^{2}+(y+Y)^{2}\right]^{-1 / 2}\left[\frac{x}{y+Y}+\frac{y+Y}{x}\right]+(y-Y)^{-1}-(y+Y)^{-1}\right\} .
\end{aligned}
$$

The positive values of square roots are to be taken in (21) and (22), and subsequently. Both equations may be written more compactly, but at the expense of some complications about the sign conventions for the roots. The last two terms of each expansion are from the two vertices at infinity, $(\infty, Y)$ and $(\infty,-Y)$. The limiting processes as $\rho_{i} \rightarrow \infty$ and $\theta_{i j} \rightarrow 0$ are performed so that $\rho_{i} \sin \theta_{i j} \equiv y-Y$ and $-y-Y$, respectively (see the Appendix). Values for $x=0$ and for $y=0$ and $\pm Y$ are especially simple, but we shall not display them here.

\subsection{Deep rectangular cracks}

The solid occupies three-dimensional space, except for the region $x>0$, $-Y<y<Y$. The required solutions are algebraically identical to those for the semi-infinite lamina, except that the sign is reversed.

\subsection{Rectangular prisms}

The solid occupies the region $-X \leqslant x \leqslant X,-Y \leqslant y \leqslant Y$. It follows from (20) that the solution takes the form

$$
\Psi(\varepsilon ; x, y)=u(\varepsilon ; X, Y)-u(\varepsilon ; X,-Y)-u(\varepsilon ;-X, Y)+u(\varepsilon ;-X,-Y),
$$

where the $u$ 's are functions of $x$ and $y$. We find, in particular, that

$$
u(6 ; X, Y)=\frac{\mu}{2}\left[(x-X)^{2}+(y-Y)^{2}\right]^{-3 / 2}\left[\left(\frac{x-X}{y-Y}\right)^{3}+\frac{3}{2}\left(\frac{x-X}{y-Y}+\frac{y-Y}{x-X}\right)+\left(\frac{y-Y}{x-X}\right)^{3}\right],
$$

$u(4 ; X, Y)=\frac{\lambda}{2}\left[(x-X)^{2}+(y-Y)^{2}\right]^{-1 / 2}\left[\frac{x-X}{y-Y}+\frac{y-Y}{x-X}\right]$.

Figure 1 gives the plot of equipotentials about an infinitely long square prism (a) for $\varepsilon=6$ and (b) for $\varepsilon=4$. An equipotential in the boundary layer approximation $[6,7]$ that $\Psi=\Psi(\nu)$ is shown for comparison. 


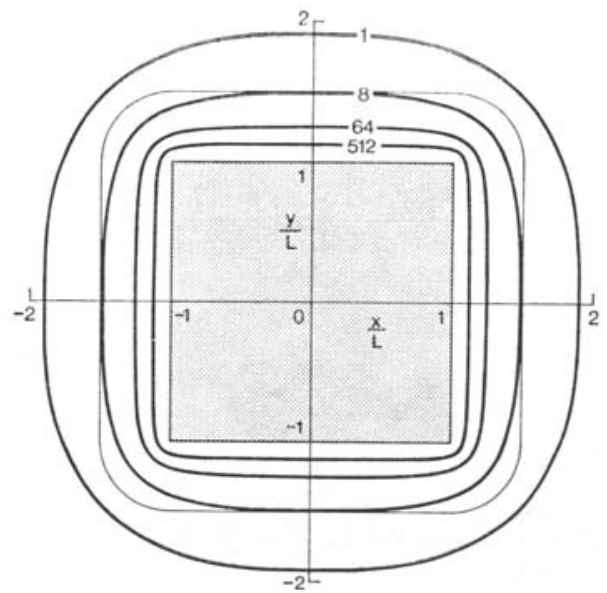

(a) $\varepsilon=6$

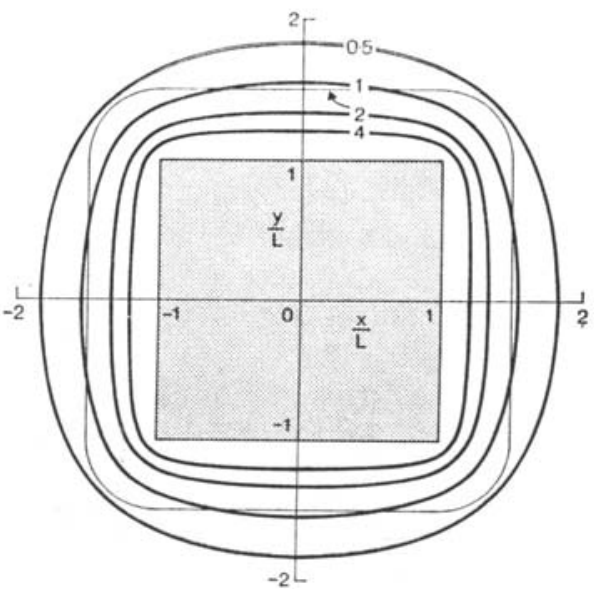

(b) $\varepsilon=4$

Fig. 1. The potential about an infinitely long square prism, $\Psi$, (a) for $\varepsilon=6$, (b) for $\varepsilon=4$. $2 L$ is the length of a side of the square. Numerals on the equipotentials denote values of (a) $-\Psi L^{3} / \mu$, (b) $-\Psi L / \lambda$. Thick curves: exact equipotentials, computed from (20). Thin curves: boundary layer approximations [6].

\subsection{Rectangular prismatic cavities}

The solid occupies three-dimensional space, except for the region $-X<x<X$, $-Y<y<Y$. The required solutions are algebraically identical to those for rectangular prisms, except that the sign is reversed. Figure 2 shows the equipotentials within an infinitely long square prismatic cavity (a) for $\varepsilon=6$ and (b) for $\varepsilon=4$. An equipotential in the boundary layer approximation is shown for comparison.

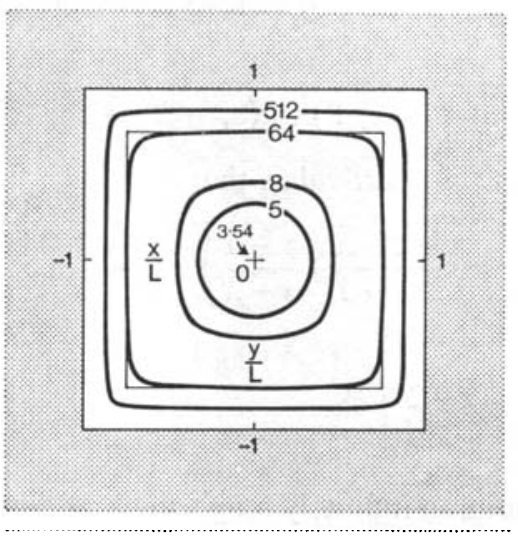

(a) $\varepsilon=6$

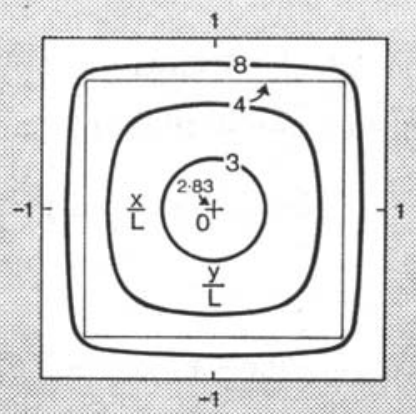

(b) $\varepsilon=4$

Fig. 2. The potential within an infinitely long square prismatic cavity. Details as for Fig. 1. 


\subsection{Regular $n$-gonal prismatic cavities}

Finally, we evaluate the potential on the axis of regular $n$-gonal prismatic cavities of infinite length, $\Psi_{n}(\varepsilon ; 0)$. It follows from (15) that

$$
\Psi_{n}(\varepsilon ; 0)=-n R^{3-\varepsilon} g\left(\varepsilon ; \frac{n-2}{2 n} \pi\right),
$$

where $R$ is the radius of the circumscribing circle of the $n$-gon. We find the particular forms

$$
\begin{aligned}
& \Psi_{n}(6 ; 0)=-n \mu R^{-3}\left(\frac{3}{2} \tan \frac{\pi}{n}+\tan ^{3} \frac{\pi}{n}\right) \\
& \Psi_{n}(4 ; 0)=-n \lambda R^{-1} \tan \frac{\pi}{n}
\end{aligned}
$$

We observe that

$$
\lim _{n \rightarrow \infty} \Psi_{n}(6 ; 0)=-\frac{3}{2} \pi \mu R^{-3}, \quad \lim _{n \rightarrow \infty} \Psi_{n}(4 ; 0)=-\pi \lambda R^{-1}
$$

The limit as $n \rightarrow \infty$ is, of course, the circular tube. These values agree exactly with the solutions for the tube [7], as they should.

The area $A$ of the regular $n$-gon is given by

$$
A=\frac{1}{2} n R^{2} \sin \frac{2 \pi}{n}
$$

so that we may eliminate $R$ between (26) and (30), and express $\Psi_{n}(\varepsilon ; 0)$ as a function of $A$ rather than $R$. We have, in particular,

$$
\begin{aligned}
& \Psi_{n}(6 ; 0)=-\mu(2 A)^{-3 / 2} n^{5 / 2} \sin ^{3 / 2} \frac{2 \pi}{n}\left(\frac{3}{2} \tan \frac{\pi}{n}+\tan ^{3} \frac{\pi}{n}\right), \\
& \Psi_{n}(4 ; 0)=-\lambda(2 A)^{-1 / 2} n^{3 / 2} \sin ^{1 / 2} \frac{2 \pi}{n} \tan \frac{\pi}{n}
\end{aligned}
$$

We may infer from (31) and (32) the dependence on $n$ of the axial potential in regular $n$-gonal cavities of equal cross-sectional area. Normalizing with respect to the tube $(n \rightarrow \infty)$, we obtain

$$
\begin{aligned}
& \frac{\Psi_{n}(6 ; 0)}{\Psi_{\infty}(6 ; 0)}=2^{-1 / 2}\left(\frac{n}{\pi}\right)^{5 / 2} \sin ^{3 / 2} \frac{2 \pi}{n}\left(\frac{1}{2} \tan \frac{\pi}{n}+\frac{1}{3} \tan ^{3} \frac{\pi}{n}\right), \\
& \frac{\Psi_{n}(4 ; 0)}{\Psi_{\infty}(4 ; 0)}=2^{-1 / 2}\left(\frac{n}{\pi}\right)^{3 / 2} \sin ^{1 / 2} \frac{2 \pi}{n} \tan \frac{\pi}{n}
\end{aligned}
$$


Table 1 presents numerical results which follow from (24) and (34). It will be seen that the axial potential converges rapidly to the value for the tube as $n$ increases. For $\varepsilon=4$, cavity geometry exerts very little influence on the axial potential: the deviation from the result for the tube is only $1.6 \%$ with $n$ as small as 4 . For $\varepsilon=6$ the deviations are some five times as large, as one might expect for shorter-range interactions.

TÁBLE 1

Axial potentials in regular $n$-gonal cavities of equal cross-section area

\begin{tabular}{|c|c|c|}
\hline$n$ & $\frac{\Psi_{n}(6 ; 0)}{\Psi_{\infty}(6 ; 0)}$ & $\frac{\Psi_{n}(4 ; 0)}{\Psi_{\infty}(4 ; 0)}$ \\
\hline $\begin{array}{r}3 \\
4 \\
5 \\
6 \\
8 \\
10 \\
12 \\
\infty\end{array}$ & $\begin{aligned} 2^{-3} \cdot 3^{19 / 4} \cdot \pi^{-5 / 2}= & 1.3220491 \\
2^{7 / 2} \cdot 3^{-1} \cdot 5 . \pi^{-5 / 2} & =1.0779014 \\
& 1.0292561 \\
2^{-1 / 2} \cdot 3^{3 / 4} \cdot 11 \cdot \pi^{-5 / 2}= & 1.0135471 \\
2^{21 / 4} \cdot 3^{-1}\left(13.2^{1 / 2}-17\right) \pi^{-5 / 2}= & 1.0041328 \\
2^{2} .3\left(58.3^{1 / 2}-99\right) \pi^{-5 / 2} & =1.0016665 \\
& 1.0007971\end{aligned}$ & $\begin{aligned} 2^{-1} \cdot 3^{9 / 4} \cdot \pi^{-3 / 2}= & 1.0635748 \\
2^{5 / 2} \cdot \pi^{-3 / 2}= & 1.0158982 \\
& 1.0059570 \\
2^{1 / 2} \cdot 3^{5 / 4} \cdot \pi^{-3 / 2}= & 1.0027479 \\
2^{15 / 4}\left(2^{1 / 2}-1\right) \pi^{-3 / 2}= & 1.0008339 \\
12.3^{1 / 2}\left(2-3^{1 / 2}\right) \pi^{-3 / 2}= & 1.0003353 \\
& 1.0001601\end{aligned}$ \\
\hline
\end{tabular}

\section{Discussion}

We conclude with some brief observations:

(a) In our treatment of the evaluation of $\Psi$ about the prism, we have not discussed the special case of points coplanar with one face of the prism. Such points yield the values $\theta_{1 j}=0$ and $\pi$ at the two relevant vertices, with the corresponding $g$ 's singular. Evaluating $\Psi$ at a point normal distance $\delta$ from the plane of the prism face, and taking the limit as $\delta \rightarrow 0$, we find that the singularities cancel, so that $\Psi$ is finite and readily evaluated.

(b) Our general results, (15) and (20), apply to a wide range of configurations. Applications include adsorptive potentials on stepped and on rough surfaces, and within cracks and near protrusions of various shapes.

(c) There seems no prospect of analogous general results for polyhedra and polyhedral cavities. Nevertheless, ad hoc use of related superposition methods yields solutions for at least some important classes of polyhedral configurations [8].

(d) A recent paper by Waldvogel [11] suggests the paucity and complication of solutions for $\varepsilon=1$. MacMillan's classical formula [5] for the Newtonial potential about a rectangular parallelepiped needs $1 \frac{1}{2}$ pages for its display alone, and Waldvogel's more concise derivation requires 3 pages. It is paradoxical that simple general results appear to be more readily available for $\varepsilon>3$ than for the 
presumably elementary case of $\varepsilon=1$. Fundamental solutions for infinite configurations do not exist for $\varepsilon \leqslant 3$, so that in this case there is no opportunity, comparable to that for $\varepsilon>3$, of generating simple general results by superposition.

(e) Peripherally to our main theme, we show in Figs. 1 and 2 one equipotential according to the elementary form of the boundary layer approximation $[6,7]$ that $\Psi=\Psi(\nu)$. The comparison of exact and boundary layer potentials afforded by these figures, and by various other results of this paper, supplements the study of this question in [7], and is fully consistent with it.

\section{Acknowledgement}

I am grateful to Dr. J. A. Barker for an important criticism of the original version of this paper.

\section{Appendix}

Coverage of the plane by double fans

In this Appendix we examine the coverage of any plane $z=$ constant through a superposition of double-wedge-shaped plane segments, which we designate "double fans". A two-dimensional discussion suffices, because the three-dimensional configurations treated in this paper are infinite in the $z$-direction; coverage of the plane by double fans is equivalent to filling three-dimensional space with threedimensional double wedges. We proceed to develop appropriate theorems.

DEFinition. An interior double fan of a polygon has as one of its apical angles one of the interior angles of the polygon.

Definition. An exterior double fan of a polygon has as its apical angles a vertical pair of exterior angles of the polygon.

THEOREM 1. The $n$ interior double fans of any n-gon, taken together, cover the whole plane, exclusive of the n-gon, $n-2$ times; and they cover the $n$-gon itself $n$ times.

We begin with the observation that the theorem is true for $n=3$. The proof consists of enumeration of the areas covered by the three double fans. The plane, exclusive of the triangle, is covered exactly once; and the triangle is covered 3 times.

Now, for $n>3$, any $n$-gon may be dissected into $n-2$ triangles, such that every vertex of every triangle is a vertex of the $n$-gon [4]. 
It follows from the foregoing result for $n=3$ that superposition of the interior double fans of all $n-2$ triangles covers the whole plane, exclusive of the $n$-gon, $n-2$ times; and that it covers the $n$-gon itself $3+(n-3)=n$ times.

Now, at every vertex of the $n$-gon, the interior angles of the component triangles, taken together, make up the interior angle of the $n$-gon. It follows that the interior double fans of all $n-2$ triangles, taken together, make up all the interior double fans of the $n$-gon.

This completes the proof of the Theorem.

THEOREM 2. The $n$ exterior double fans of any n-gon, taken together, cover the whole plane, exclusive of the $n$-gon, twice; and they leave the $n$-gon itself uncovered.

At any vertex of the $n$-gon, the interior double fan and the exterior double fan, taken together, cover the total plane. Summing over all $n$ vertices, then,

$$
\sum_{i=1}^{n}(\text { exterior double fan })_{i}=n(\text { total plane })-\sum_{i=1}^{n}(\text { interior double fan })_{i} .
$$

But Theorem 1 asserts

$$
\sum_{i=1}^{n}(\text { interior double fan })_{i}=(n-2)(\text { total plane })+2(n \text {-gon }) .
$$

Eliminating $\sum_{i=1}^{n}($ interior double fan) $i$ between (A.1) and (A.2) yields

$$
\sum_{i=1}^{n} \text { (exterior double fan) }{ }_{i}=2 \text { (total plane }-n \text {-gon). }
$$

This establishes the Theorem.

The dissection into $n-2$ triangles holds not only for convex $n$-gons, but also for non-convex ones [4]; so that Theorems 1 and 2 apply to both convex and nonconvex $n$-gons. Non-convex $n$-gons have at least one reflex interior angle. In this connection we note that the double fan associated with a reflex angle contained between the lines $\theta=2 \pi-\beta(\pi<\beta<2 \pi)$ and $\theta=2 \pi$ consists of double coverage of the regions $2 \pi-\beta \leqslant \theta \leqslant \pi, 3 \pi-\beta \leqslant \theta \leqslant 2 \pi$, together with single coverage of the regions $0 \leqslant \theta \leqslant 2 \pi-\beta, \pi \leqslant \theta \leqslant 3 \pi-\beta$.

Theorems 1 and 2 apply also to $n$-gons with two or more vertices at infinity. Such $n$-gons present no special features except that, in respect of equations such as (17) and (18), care must be exercised in proceeding to the limit as $\rho_{i} \rightarrow \infty$ and $\theta_{i j} \rightarrow 0$. 


\section{REFERENCES}

[1] J. Clifford, "Properties of water in capillaries and thin films", in Water, a comprehensive treatise (ed. F. Franks) (Plenum, New York, 1975), Vol. 5, pp. 75-132.

[2] A. Erđélyi, W. Magnus, F. Oberhettinger and F. G. Tricomi, Higher transcendental functions (McGraw-Hill, New York, 1953), Vol. I, p. 160, equation (33).

[3] D. H. Everett, "The effect of adsorption on the interaction between solid particles", Pure and Appl. Chem. 48 (1976), 419-425.

[4] H. G. Forder, The foundations of Euclidean geometry (Dover, New York, 1958), pp. 244-254.

[5] W. D. MacMillan, The theory of the potential (Dover, New York, 1958), pp. 72-80.

[6] J. R. Philip, "Unitary approach to capillary condensation and adsorption", J. Chem. Phys. 66 (1977), 5069-5075.

[7] J. R. Philip, "Adsorption and geometry: the boundary layer approximation", J. Chem. Phys. 67 (1977), 1732-1741.

[8] J. R. Philip, "Inverse power law potentials in rectangular configurations", $Z$. angew. Math. Phys. (in press).

[9] A. Sheludko, "Thin liquid films", Adv. Coll. Interface Sci. 1 (1967), 390-464.

[10] W. A. Steele and G. D. Halsey, Jr., "The interaction of gas molecules with capillary and crystal lattice surfaces", J. Phys. Chem. 59 (1955), 57-65.

[11] J. Waldvogel, "The Newtonian potential of a homogeneous cube", Z. angew. Math. Phys. 27 (1976), 867-871.

CSIRO Division of Environmental Mechanics

P.O. Box 821

Canberra City, A.C.T. 2601

Australia 